\title{
Thermal-Boundary-Layer Response to Convected Far-Field Fluid Temperature Changes
}

\author{
Hongwei Li \\ School of Mechanical Engineering, \\ Purdue University, \\ West Lafayette, IN 47907 \\ M. Razi Nalim ${ }^{1}$ \\ Department of Mechanical Engineering, \\ Indiana University-Purdue University \\ Indianapolis, \\ Indianapolis, IN 46202 \\ e-mail: mnalim@iupui.edu
}

Fluid flows of varying temperature occur in heat exchangers, nuclear reactors, nonsteady-flow devices, and combustion engines, among other applications with heat transfer processes that influence energy conversion efficiency. A general numerical method was developed with the capability to predict the transient laminar thermalboundary-layer response for similar or nonsimilar flow and thermal behaviors. The method was tested for the step change in the far-field flow temperature of a twodimensional semi-infinite flat plate with steady hydrodynamic boundary layer and constant wall temperature assumptions. Changes in the magnitude and sign of the fluid-wall temperature difference were considered, including flow with no initial temperature difference and built-up thermal boundary layer. The equations for momentum and energy were solved based on the Keller-box finite-difference method. The accuracy of the method was verified by comparing with related transient solutions, the steady-state solution, and by grid independence tests. The existence of a similarity solution is shown for a step change in the far-field temperature and is verified by the computed general solution. Transient heat transfer correlations are presented, which indicate that both magnitude and direction of heat transfer can be significantly different from predictions by quasisteady models commonly used. The deviation is greater and lasts longer for large Prandtl number fluids. [DOI: 10.1115/1.2953239]

Keywords: thermal boundary layer, transient heat transfer, nonsteady flow, wave rotor

\section{Introduction}

Nonsteady heat convection occurs in many natural and engineered systems. Of particular interest is the flow of gases of varying temperatures in energy conversion devices. In such practical applications, classical steady heat transfer correlations are widely used, with an assumption of quasisteadiness. This may cause significant heat transfer prediction errors in both direction and magnitude due to the transient thermal response. For example, Annand and Pinfold [1] measured the heat transfer rate to the internal surface of an operating internal combustion engine and found that the heat transfer rate is out of phase with the bulk-gas wall temperature difference. Kornhauser and Smith [2] derived a complex Nusselt number for the in-cylinder heat transfer during the compression and expansion to account for the unsteady thermal effect on heat transfer. These studies of gross heat transfer did not examine details of the boundary-layer transients.

Accurate knowledge of the time-varying thermal response is necessary in the design and transient operation of unsteady systems, such as regeneration heat exchangers, nuclear reactors, combustion engines, shock tubes, and wave rotors. Significant fuel efficiency and emission reduction benefits are offered by machines that exploit nonsteady flow, such as wave rotors and pulsed combustion devices [3-5]. These machines require an accurate prediction of heat transfer in passages with varying gas temperatures.

The convection of nonuniform fluid with a moving temperature-step contact surface has important engineering application in the channels of regenerative heat exchangers and wave rotors. These machines are typically constructed as rotating drums

\footnotetext{
${ }^{1}$ Corresponding author.

Contributed by the Heat Transfer Division of ASME for publication in the Journal of Heat Transfer. Manuscript received August 7, 2007; final manuscript received December 23, 2007; published online August 7, 2008. Review conducted by Louis C. Burmeister. Paper presented at the 2007 ASME International Mechanical Engineering Congress (IMECE2007), Seattle, WA, November 10-16, 2007.
}

with a number of flow channels that admit different gases alternately. The wave rotor, in particular, has been shown to have potential to improve aircraft and gas turbine energy efficiency and to reduce emission of greenhouse gases by $10-25 \%$. Regenerative heat exchangers also have important energy conversion benefits [6].

Using pressure waves like a shock tube [7] and possibly confined pressure-gain combustion, the wave rotor affects direct pressure exchange and work transfer between inert or reacting fluids [8]. By rotation, each channel is periodically charged from or discharged to inlet and outlet partial annular ports. The alignment of the rotating channel with an inlet port creates a pressure wave that travels rapidly into the gas inside the channel, equalizing pressure and velocity. More slowly, the channel ingests fresh gas flow that has a different composition and temperature, but now shares the same velocity as the gas inside the channel.

The fundamental behavior of such transient thermal problems can be well modeled as an external boundary-layer problem on a two dimensional (2D) semi-infinite flat plate using standard boundary-layer assumptions. The present study is limited to laminar flow conditions with a steady-flow velocity field.

Changes in a thermal boundary layer can be induced through different actions, such as imposing a heat flux at the wall [9-11], change in wall surface temperature [12], or change in fluid temperature $[13,14]$. This paper focuses on the transient laminar thermal boundary layer due to a moving incompressible fluid of varying temperatures. A general methodology was developed for the simulation of unsteady thermal-boundary-layer behavior subject to uniform or nonuniform flow and thermal boundary conditions [15]. The method is illustrated for the cases of an initial steady state subject to a sudden step temperature change as the fluid of different temperature sweeps over the plate, with unchanged velocity. As the contact surface between the two fluids travels down the plate, the upstream boundary layer asymptotically approaches a new steady state.

The cases studied here include flows with and without an initial 
thermal boundary layer, subject to a step change in far-field fluid temperature. A range of Prandtl number is considered to illustrate the effect of the relative size of the thermal boundary layer, which must be accommodated in the numerical method.

The governing incompressible laminar 2D unsteady-boundarylayer equations were solved using Keller's box method [16]. This is an implicit finite-difference method with second-order accuracy for both spatial and temporal variables and can be improved to higher orders of accuracy [17]. The method has been applied to forced-convection problems such as two-dimensional [18] and three-dimensional steady flows $[19,20]$ as well as twodimensional time-dependent flow [21], in addition to thermalboundary-layer problems [22]. It has also been applied to freeconvection flow [23] and combined forced and free-convection flow [24]. According to the Keller-box method, the high-order differential equations are rewritten as a system of first-order equations by introducing new dependent variables. The system is then linearized using Newton's method, and the resulting system is solved effectively by using the block-elimination method [25]. A marching procedure along the flow direction $(x)$ at each time step requires iteration at each $x$-station until some convergence criterion is satisfied.

\section{General Equations}

A steady hydrodynamic boundary layer is established, the fluid moving with constant velocity $u_{\infty}$ far from the plate. At time $t$ $<0$, the bulk fluid temperature is constant at $T_{\infty 1}$. In the general case when $T_{\infty 1}$ is different from the plate temperature $T_{w}$, a steady initial thermal boundary layer is also established. At time $t=0$, the incoming far-field fluid temperature at the leading edge is suddenly changed to $T_{\infty 2}$. For $t>0$, the far-field fluid at $T_{\infty 2}$ is convected down the plate at speed $u_{\infty}$. The appropriate far-field temperature boundary condition is applied on each side of the contact surface between the fluids, while the plate temperature remains constant. The fluid is assumed incompressible, and the density and other fluid properties are assumed constant, so that the 2D boundary-layer equations are uncoupled. Viscous dissipation is neglected and the Eckert number is assumed small due to low fluid velocity [26].

The boundary-layer continuity and momentum equations for the 2D laminar incompressible flow have the following timesteady form:

$$
\begin{gathered}
\frac{\partial u}{\partial x}+\frac{\partial v}{\partial y}=0 \\
u \frac{\partial u}{\partial x}+v \frac{\partial u}{\partial y}=\nu \frac{\partial^{2} u}{\partial y^{2}}
\end{gathered}
$$

The boundary conditions applied to Eqs. (1) and (2) are

$$
u(x, 0)=v(x, 0)=0 \quad \text { and } \quad u(x, \infty)=u_{\infty}
$$

The time-dependent energy equation

$$
\frac{\partial T}{\partial t}+u \frac{\partial T}{\partial x}+v \frac{\partial T}{\partial y}=\alpha \frac{\partial^{2} T}{\partial y^{2}}
$$

is subject to the initial condition

$$
T(x, y>0, t<0)=T_{\infty 1}
$$

and boundary conditions

$$
\begin{gathered}
T\left(x \leqslant u_{\infty} t, y \rightarrow \infty, t \geqslant 0\right)=T_{\infty 2} \\
T\left(x>u_{\infty} t, y \rightarrow \infty, t \geqslant 0\right)=T_{\infty 1} \\
T(x, y=0, t)=T_{w}
\end{gathered}
$$

where $T_{\infty 2}$ can be equal to, greater than, or less than $T_{\infty 1}$. Equations (2) and (4) are nondimensionalized as

$$
\begin{gathered}
\bar{U} \frac{\partial \bar{U}}{\partial X}+\bar{V} \frac{\partial \bar{U}}{\partial Y}=\frac{\partial^{2} \bar{U}}{\partial Y^{2}} \\
\frac{\partial \theta}{\partial \tau}+\bar{U} \frac{\partial \theta}{\partial X}+\bar{V} \frac{\partial \theta}{\partial Y}=\frac{1}{\operatorname{Pr}} \frac{\partial^{2} \theta}{\partial Y^{2}}
\end{gathered}
$$

where

$$
\begin{gathered}
X=\frac{x}{L}, \quad Y=\frac{y}{L} \sqrt{\operatorname{Re}_{L}}, \quad \bar{U}=\frac{u}{u_{\infty}}, \quad \bar{V}=\frac{v}{u_{\infty}} \sqrt{\operatorname{Re}_{L}} \\
\theta=\frac{T-T_{\infty}}{T_{w}-T_{\infty}}, \quad \tau=\frac{t u_{\infty}}{L}
\end{gathered}
$$

$T_{\infty}$ is $T_{\infty 1}$ if a thermal boundary layer exists initially. $T_{\infty}$ is $T_{\infty 2}$ if $T_{\infty 1}=T_{w}$.

Using the standard definition of the stream function, $\psi(\bar{U}$ $=\partial \psi / \partial Y, \bar{V}=-\partial \psi / \partial X)$, the boundary layer similarity transformation is introduced,

$$
\eta=\frac{Y}{\sqrt{X}}, \quad f(X, \eta)=\frac{\psi}{\sqrt{X}}
$$

where $\eta$ is the similarity variable and $f$ is the nondimensional reduced stream function. Unlike for some steady boundary layers with constant velocity or power-law velocity variation, the $X$-dependence is not eliminated by this step for a general far-field temperature variation, only reduced.

The nondimensional momentum equation and the timedependent energy equation can be written as

$$
\begin{gathered}
f^{\prime \prime \prime}+\frac{1}{2} f f^{\prime \prime}=X\left[f^{\prime} \frac{\partial f^{\prime}}{\partial X}-f^{\prime \prime} \frac{\partial f}{\partial X}\right] \\
\frac{1}{\operatorname{Pr}} \theta^{\prime \prime}+\frac{1}{2} f \theta^{\prime}=X\left[f^{\prime} \frac{\partial \theta}{\partial X}-\theta^{\prime} \frac{\partial f}{\partial X}+\frac{\partial \theta}{\partial \tau}\right]
\end{gathered}
$$

The number of prime marks on $f$ and $\theta$ denotes the degree of differentiation with respect to $\eta$, the first derivative being $f^{\prime}$. The boundary conditions for the steady momentum equation are rewritten as

$$
f(X, 0)=f^{\prime}(X, 0)=0, \quad f^{\prime}(X, \infty)=1
$$

With the above definition of $\theta$, when the initial fluid temperature is different from the wall temperature, the boundary conditions are

$$
\begin{gathered}
\theta(X \leqslant \tau, \infty, \tau \geqslant 0)=\frac{T_{\infty 2}-T_{\infty 1}}{T_{w}-T_{\infty 1}}=R \\
\theta(X \geqslant \tau, \infty, \tau \geqslant 0)=0, \quad \theta(X, 0, \tau)=1
\end{gathered}
$$

When the initial fluid and wall temperatures are equal, the boundary conditions become

$$
\begin{aligned}
& \theta(X \leqslant \tau, \infty, \tau \geqslant 0)=0 \\
& \theta(X>\tau, \infty, \tau \geqslant 0)=1
\end{aligned}
$$

\section{Regional Self-Similar Solution}

Although Eqs. (10) and (11) indicate the solution dependency on $X$ and $\tau$, the thermal boundary layer can be shown to be selfsimilar with respect to a dimensionless ratio, $\tau^{+}=\tau / X=u_{\infty} t / x$, for any far-field temperature variation that is purely convected at a constant $u_{\infty}$, given a uniform wall boundary condition. The solutions with respect to $\eta$ collapse to a single curve for a given value of $\tau^{+}$, which, in the case of a step change in far-field temperature, represents the distance traveled by the contact surface from the leading edge, relative to the distance $x$ of any location of interest. 


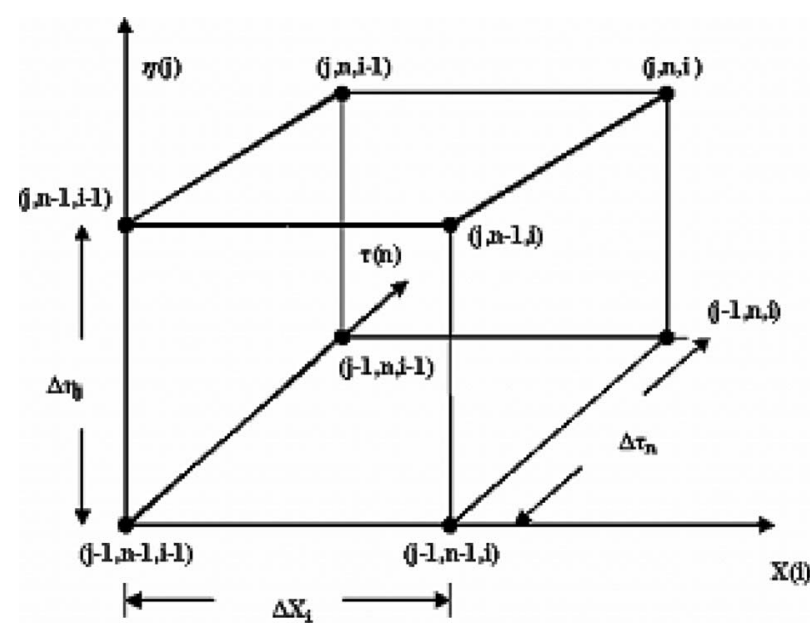

Fig. 1 Finite-difference grid for the Keller-box scheme

The elapsed time after the contact surface passes a given location is represented by $\tau^{+}-1$. This self-similar character can be shown by introducing a similar transformation with the definition of $\tau^{+}$, reducing Eqs. (2) and (4) to

$$
f^{\prime \prime \prime}+\frac{1}{2} f f^{\prime \prime}=0
$$

and

$$
\frac{1}{\operatorname{Pr}} \theta^{\prime \prime}+\frac{1}{2} f \theta^{\prime}=\frac{\partial \theta}{\partial \tau^{+}}\left(1-f^{\prime} \tau^{+}\right)
$$

The transformation in Eqs. (15) and (16) now eliminates the $X$-dependency in the equations. If the boundary conditions are also independent of $X$, the solutions will be functions of $\eta$ and $\tau^{+}$ only. In the undisturbed region with $\tau^{+}<1$, the self-similar boundary-layer solution remains as a function of $\eta$ alone.

Note that when the temporal coefficient $\left(1-f^{\prime} \tau^{+}\right)$changes sign from positive for locations close to the plate (where $f^{\prime} \ll 1$ ) to negative for locations close to the upper boundary layer, a combination of different finite-difference schemes is required. Equations (15) and (16) have been solved for other $X$-independent boundary conditions, using appropriate numerical methods [12].

\section{Numerical Discretization and Method Validation}

In this study, a general methodology is presented for problems with similar or nonsimilar flow and thermal behaviors. The similarity character for the special case above is verified. The Kellerbox numerical discretization is based on the idea of expressing all functions and derivatives in terms of quantities at the corners of one computational block. All derivatives are approximated by simple centered-difference and two-point averages using only values at the corners of the three-dimensional box (or rectangle mesh) [16]. The box schematic is shown in Fig. 1, with $X_{i}=X_{i-1}$ $+\Delta X_{i}, \tau_{n}=\tau_{n-1}+\Delta \tau_{n}$, and $\eta_{j}=\eta_{j-1}+\Delta \eta_{j}$. The spacings $\Delta X_{i}, \Delta \eta_{j}$, and $\Delta \tau_{n}$ are independently variable.

New dependent variables $g, h$, and $e$ are introduced to eliminate all higher-order derivatives, and a system of first-order partial differential equations is formed. The transformed Eqs. (10) and (11) are rewritten as

$$
\begin{aligned}
& g=\frac{\partial f}{\partial \eta}=f^{\prime} \\
& h=\frac{\partial g}{\partial \eta}=g^{\prime}
\end{aligned}
$$

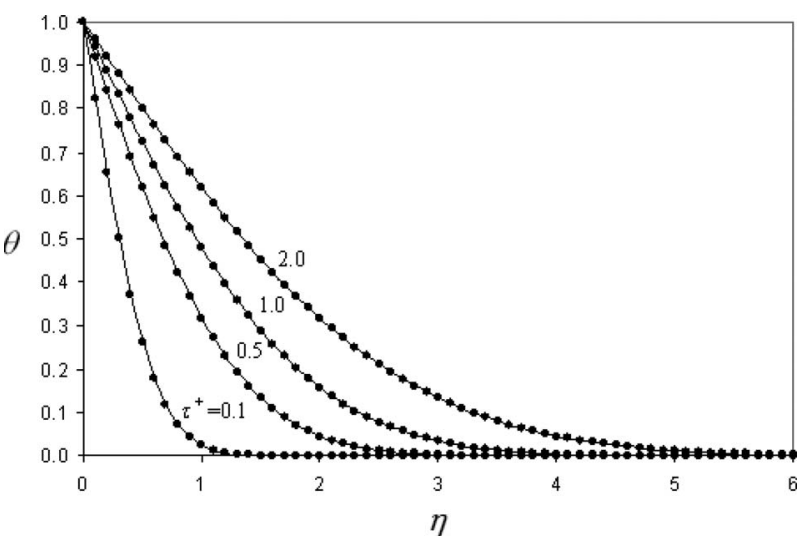

Fig. 2 Present-method numerical (dots) and analytical (line) solutions of the heat equation for a range of $\tau^{+}$

$$
\begin{gathered}
e=\frac{\partial \theta}{\partial \eta}=\theta^{\prime} \\
h^{\prime}+\frac{1}{2} f h=X\left(g \frac{\partial g}{\partial X}-h \frac{\partial f}{\partial X}\right) \\
\frac{1}{\operatorname{Pr}} e^{\prime}+\frac{1}{2} f e=X\left(g \frac{\partial \theta}{\partial X}-e \frac{\partial f}{\partial X}+\frac{\partial \theta}{\partial \tau}\right)
\end{gathered}
$$

The first-order partial differential equations (Eqs. (17)-(19)) are discretized about the point $\left(x_{i}, \eta_{j-1 / 2}\right)$, Eq. (20) is discretized at $\left(x_{i-1 / 2}, \eta_{j-1 / 2}\right)$, and Eq. $(21)$ is discretized at $\left(x_{i-1 / 2}, \eta_{j-1 / 2}, \tau_{n-1 / 2}\right)$.

The transformed laminar boundary-layer thickness is nominally constant; the computation domain extends to $\eta_{\max }=8$, and the velocity attains 0.999 of its freestream value around $\eta=6$. The velocity and temperature field were calculated using uniform grid spacings $\Delta \tau=0.001, \Delta X=0.001$, and $\Delta \eta=0.01$ for smooth highresolution solutions. Grid sensitivity was examined by choosing successively finer grids, and they showed no significant improvement on the solution accuracy.

The time accuracy of the described methodology and its computer coding were validated by a comparison with the known analytical solution of a problem with a sudden temperature change of an infinite flat plate. At time $t=0$, the plate temperature has a sudden change, while the far-field fluid temperature remains constant. This problem is mathematically expressed as the heat diffusion equation

$$
\frac{\partial \theta}{\partial t}=a \frac{\partial^{2} \theta}{\partial y^{2}}
$$

with initial and boundary conditions

$$
\begin{gathered}
\theta=\theta_{w}=1 \quad \text { at } \quad y=0, \quad t \geqslant 0 \\
\theta=\theta_{\infty}=0 \text { at } y \rightarrow \infty, \quad t \geqslant 0 \\
\theta=\theta_{\infty}=0 \quad \text { at } \quad 0 \leqslant y<\infty, \quad t<0
\end{gathered}
$$

The analytical solution [27] is

$$
\theta=1-\operatorname{erf}\left(\frac{y}{\sqrt{4 a t}}\right)=1-\operatorname{erf}\left(\eta \sqrt{\frac{X \operatorname{Pr}}{4 \tau}}\right)
$$

using the error function

$$
\operatorname{erf}(\xi)=\frac{2}{\sqrt{\pi}} \int_{0}^{\xi} e^{-z^{2}} d z
$$

The numerical solution for $\operatorname{Pr}=1$, shown in Fig. 2, matches the analytical solution quite well, using $\Delta \eta=0.1$ and $\Delta \tau=0.001$. 


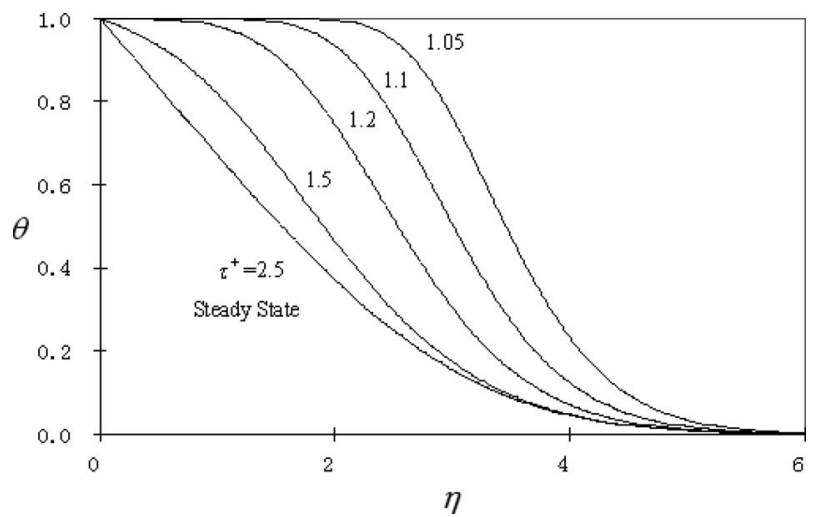

Fig. 3 Temperature response to the step change in incoming fluid temperature, with no initial thermal boundary layer

\section{Solution for Nonuniform Convected Flow}

The convected nonuniform fluid or moving contact-surface problem is governed by a different mechanism from previous transient heat transfer analyses for uniform plate or fluid temperature changes [13]. The far-field temperature disturbance is convected with freestream velocity and diffuses across the boundary layer $[28,29]$ only after the disturbance travels to a given position, so that locally $\tau^{+} \geqslant 1$.

The final steady-state solution was separately calculated through the steady energy equation, so as to provide an independent verification of the asymptotic approach of the transient solution to the steady solution for large $\tau^{+}$. When $\operatorname{Pr}=1.0$, the steady momentum equation serves as an additional verification, as the numerical solution of $1-f^{\prime}$ and $\theta$ are then identical.

Qualitatively, there are four possible nontrivial transitions: (1) no initial thermal boundary layer, (2) increase or (3) decrease in magnitude of fluid-plate temperature difference, but with the same direction of heat transfer, and (4) change in direction of fluid-plate temperature difference and heat transfer. Case (2) is not presented for brevity.

The transient temperature profile is shown in Fig. 3 for a step change in incoming fluid temperature when there is no initial thermal boundary layer and $\operatorname{Pr}=1.0$ for increasing $\tau^{+}>1$. A steep temperature gradient occurs in the middle range of the developed thermal boundary layer, but the temperature gradient at the wall does not change significantly until after $\tau^{+}$exceeds 1.2 . The final steady solution computed directly from the steady form of the equations is indistinguishable from the solution computed from the unsteady equations for $\tau^{+}=2.5$. The solution was computed at different $X$ locations away from the leading edge and was verified to be similar for given $\tau^{+}>1$.

It is more general for the initial fluid and wall temperatures to differ, with an initial existing thermal boundary layer subject to a step change in incoming fluid temperature. The change in temperature difference is expressed as a ratio $R$, as defined in Eq. (13). The transient thermal response for a step change $R=0.5$ is shown in Fig. 4. For the early transient states, the temperature changes rapidly in the outer region of the boundary layer, asymptotic to the changed upper boundary value, but near the wall remains asymptotically close to the initial steady state. A temperature extremum appears at some location along the $\eta$ direction, and bidirectional heat conduction exists temporarily within the boundary layer. As time passes, the extremum moves toward the wall and disappears; thereafter heat transfer is unidirectional and the temperature ultimately reaches a new steady state.

At the wall, the direction of heat transfer remains the same, but the magnitude changes; the conventional heat transfer coefficient as defined by the far-field temperature remains positive.

The temperature history for a step change of incoming fluid

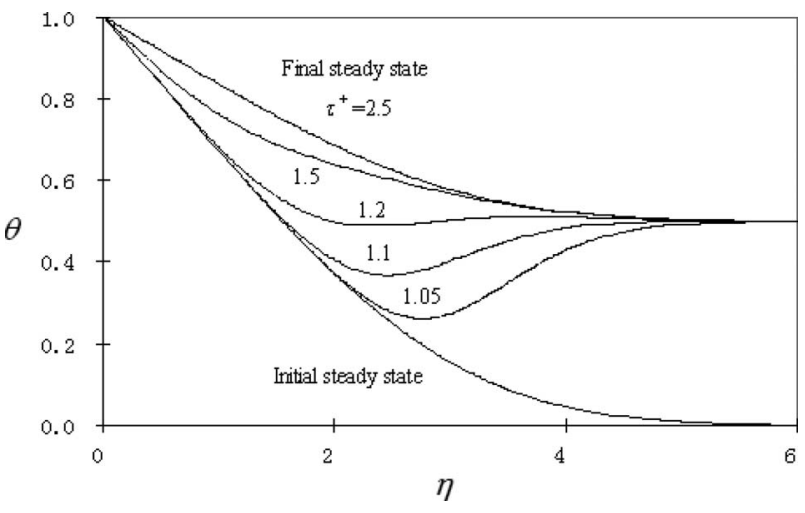

Fig. 4 Temperature response to a step change in incoming fluid temperature, with an initial thermal boundary layer $(\boldsymbol{R}$ $=0.5$ )

temperature, with $R=2.0$, is shown in Fig. 5, so that the fluid-wall temperature difference changes sign. Similar to the $R=0.5$ case, the transient temperature profile has an extremum at some $\eta$ but becomes monotone again in the final steady state as the extremum reaches the wall and disappears. In this case, both the magnitude and direction of the heat transfer change, and the conventional heat transfer coefficient based on the local far-field temperature becomes temporarily negative.

The local Nusselt number is defined as $\mathrm{Nu}_{x}=q_{w}(x) x / \lambda\left(T_{w}\right.$ $\left.-T_{\infty 2}\right)$, where the heat flux is expressed as $q_{w}(x)=-\left.\lambda(\partial T / \partial y)\right|_{y=0}$. After nondimensionalization and application of the similarity transformation, the local Nusselt number is expressed as

$$
\mathrm{Nu}_{x} \operatorname{Re}_{x}^{-1 / 2}=-\frac{\partial \theta}{\partial \eta}(x, 0, \tau) \frac{T_{w}-T_{\infty 1}}{T_{w}-T_{\infty 2}}=-\frac{e(x, 0, \tau)}{1-R}
$$

This common definition of Nu using the local far-field temperature $T_{\infty 2}$ anticipates a Newtonian heat transfer direction, which may sometimes be negated by the temperature gradient at the wall during the transient. Thus for $R=2.0$, the initial heat transfer direction is dominated by the prior far-field temperature, and $\mathrm{Nu}$ is initially negative.

The relative thickness of momentum and thermal boundary layers in the steady parallel laminar flow is related to the Prandtl number by $\delta / \delta_{t}=\operatorname{Pr}^{1 / 3}$ [26] for large Pr. For a given flow condition, Pr has a direct effect on temperature profile. In the transient boundary layer, $\operatorname{Pr}$ also determines the response time of the change as it expresses the rate of thermal diffusion relative to the momentum diffusion. The transition of the local Nusselt number

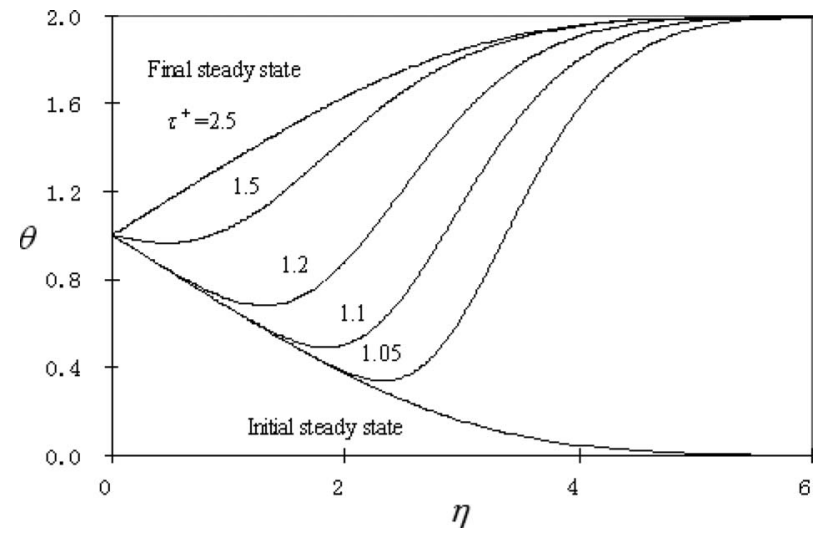

Fig. 5 Temperature response to a step change in incoming fluid temperature with an initial thermal boundary layer $(\boldsymbol{R}$ =2.0) 


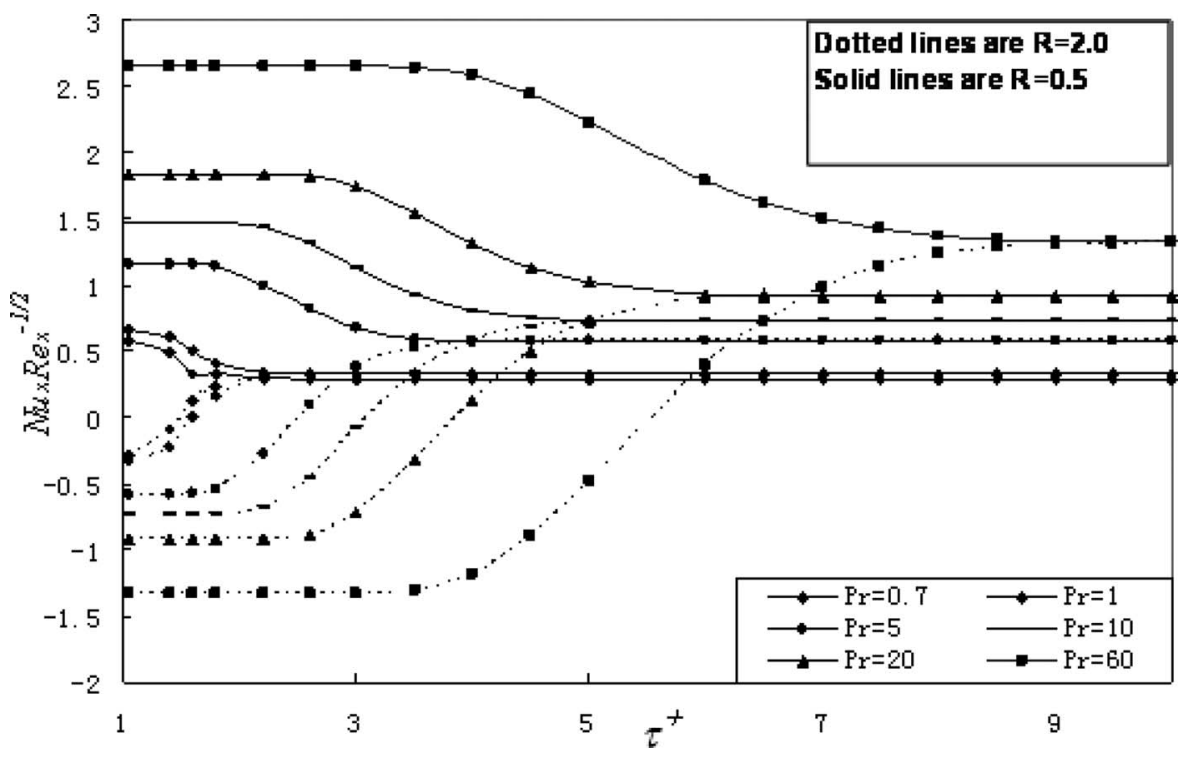

Fig. 6 Transitions of the local Nusselt number for step changes $R=0.5$ and $R=2.0$ at different $\mathrm{Pr}$

is shown in Fig. 6 for incoming fluid temperature-step changes of $R=0.5$ and $R=2.0$ for a range of Prandtl numbers. For $R=0.5$, the local Nusselt number decreases over time until the final steady state while remaining positive. This corresponds to the change in temperature gradient seen in Fig. 4. For $R=2.0$, the local Nusselt number is negative initially as the heat transfer rate at the wall remains in the initial direction, but the wall-fluid temperature difference that defines $\mathrm{Nu}$ changes sign. For the $\mathrm{Pr}=1$ case of Fig. 5, the location of the temperature extremum reaches the wall at approximately $\tau^{+}=1.6$, whereupon the direction of heat transfer at the wall changes sign. Thus, there is a period of time for which the heat transfer predicted here differs in direction (not just in magnitude) from any quasisteady model that follows the classical Newton cooling law. There will be a serious qualitative prediction error if the steady heat transfer correlation is applied immediately after the far-field temperature changes, especially for larger Pr.

This error may be important in unsteady-flow device models, such as a widely used quasi-one-dimensional model for wave rotor flows [30]. In this code, the steady-state Reynolds-Colburn analogy is invoked to model heat transfer based on the local bulkgas temperature without regard to the changing temperature history of the flow.

The effect of the Prandtl number on local Nusselt numbers is shown in Fig. 6. For larger Pr, the transition from an initial to a final steady state occurs later and takes longer than that for smaller Pr due to the smaller diffusivity of heat relative to momentum. A range of $\mathrm{Pr}$ is explored from 0.7 for typical gases and air to 60 for some oils, which includes many useful fluids such as liquid water $(\operatorname{Pr} \approx 5)$ and water vapor $(\operatorname{Pr} \approx 1)$. For $\operatorname{Pr}=0.7$, the wall heat transfer change occurs over a range of $\tau^{+}$from near 1 to 2.5 , whereas for $\operatorname{Pr}=60$, the change occurs over a range of $\tau^{+}$from 4 to 9 approximately. The larger Pr also results in a higher absolute value of the local Nusselt number. The $\mathrm{Nu}$ curves for $R$ $=0.5$ and $R=2.0$ asymptotically reach the same value, as the final temperature profile only depends on the final temperatures and fluid properties.

\section{Conclusions}

The transient thermal boundary-layer response due to a convected nonuniform temperature fluid was numerically investigated for an incompressible laminar flow under the assumption of a steady hydraulic boundary layer on a semi-infinite plate. Far-field temperature changes that result in changes in the magnitude and/or direction of the fluid-plate heat transfer were considered, including the case where no thermal boundary layer exists initially. Unlike the problems of uniform change of fluid or plate temperature, the boundary layer experiences no changes until the convected far-field variation or contact surface arrives at a given plate location. A time-accurate boundary-layer flow and temperature computation methodology was developed, verified against some analytical solutions and prior work, and then applied to predict the boundary-layer temperature profiles in response to a convected step change in fluid temperature. The analysis of the local instantaneous Nusselt number shows that the use of steadystate heat transfer correlations in transient thermal states can cause both magnitude and direction of heat transfer rate to be incorrectly predicted during the transition. The response time and thermal boundary-layer thickness are correlated with the fluid Prandtl number.

It is anticipated that the numerical methodology developed here will be extended to compressible and turbulent flows. The errors related to quasisteady assumptions may be less significant for turbulent flow in gases but must be quantified for either justification or relaxation of this assumption. Practical nonsteady devices such as the wave rotor subject to complex temperature variations of a flowing gas may then be modeled with a realistic transient heat transfer prediction. This will enable important technologies to be improved for energy conversion efficiency and other benefits.

\section{Acknowledgment}

H.L. was partially supported by a fellowship from Indiana University-Purdue University Indianapolis (IUPUI) and by the Indiana 21st Century Fund for Research and Technology managed by Rolls Royce Liberty Works during this effort. The advice of Dr. C. Merkle is gratefully acknowledged.

\section{Nomenclature}

$$
\begin{aligned}
e & =\text { dependent differential variable } \\
f & =\text { nondimensional reduced stream function } \\
g & =\text { dependent differential variable } \\
h & =\text { dependent differential variable } \\
L & =\text { characteristic length } \\
\mathrm{Nu} & =\text { Nusselt number } \\
\operatorname{Pr} & =\text { Prandtl number } \\
q & =\text { heat flux }
\end{aligned}
$$




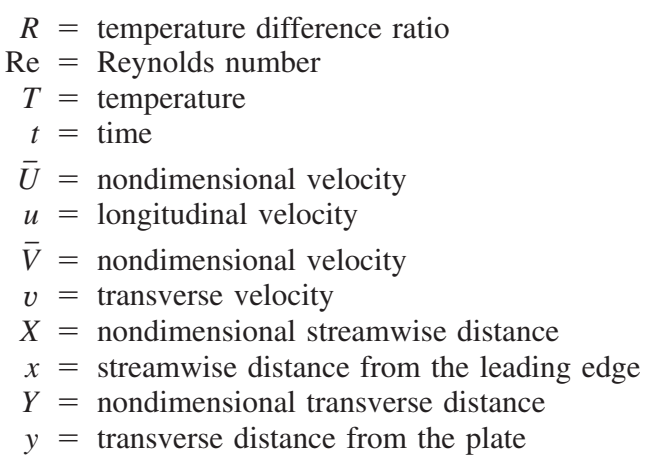

Greek Symbols

$\begin{aligned} \alpha & =\text { thermal diffusivity } \\ \eta & =\text { similarity variable } \\ \theta & =\text { dimensionless temperature } \\ \lambda & =\text { thermal conductivity } \\ \tau & =\text { dimensionless time } \\ \tau^{+} & =\text {dimensionless ratio of } \tau \text { to } X \\ \nu & =\text { kinematic viscosity } \\ \psi & =\text { stream function }\end{aligned}$

Subscripts

$i=$ grid index in the longitudinal direction

$j=$ grid index in the transverse direction

$n=$ grid index in the temporal direction

$w=$ wall

$\infty=$ far-field flow

\section{References}

[1] Annand, W. J. D., and Pinfold, D., 1980, "Heat Transfer in the Cylinder of a Motored Reciprocating Engine," SAE Paper No. 800457.

[2] Kornhauser, A. A., and Smith, J. L. Jr., 1994, "Application of a Complex Nusselt Number to Heat Transfer During Compression and Expansion," ASME J. Heat Transfer, 116, pp. 536-542.

[3] Wilson, J., and Paxson, D. E., 1996, "Wave Rotor Optimization for Gas Turbine Engine Topping Cycles," J. Propul. Power, 12, pp. 778-785.

[4] Nalim, M. R., and Paxson, D. E., 1997, "A Numerical Investigation of Premixed Combustion in Wave Rotors," ASME J. Eng. Gas Turbines Power, 119, pp. 668-675.

[5] Kailasanath, K., 2002, "Recent Developments in the Research on Pulse Detonation Engines," J. Propul. Power, 18, pp. 77-83.

[6] Klein, H., and Eigenberger, G., 2001, "Approximate Solutions for Metallic Regenerative Heat Exchanger,' Int. J. Heat Mass Transfer, 44, pp. 3553-3563.

[7] Fujii, N., Koshi, M., Ando, H., and Asaba, T., 1979, "Evaluation of BoundaryLayer Effects in Shock-Tube Studies of Chemical Kinetics," Int. J. Chem. Kinet., 11, pp. 285-304.
[8] Nalim, M. R., 2000, "Longitudinally Stratified Combustion in Wave Rotors," J. Propul. Power, 16, pp. 1060-1068.

[9] Rebay, M., and Padet, J., 2005, "Parametric Study of Unsteady Forced Convection With Pressure Gradient," Int. J. Eng. Sci., 43, pp. 655-667.

[10] Harris, S. D., Ingham, D. B., and Pop, I., 2001, "Transient Boundary-Layer Heat Transfer From a Flat Plate Subjected to a Sudden Change in Heat Flux," Eur. J. Mech. B/Fluids, 20, pp. 187-204.

[11] Polidori, G., Lachi, M., and Padet, J., 1998, "Unsteady Convective Heat Transfer on a Semi-Infinite Flat Surface Impulsively Heated," Int. Commun. Heat Mass Transfer, 25, pp. 33-42.

[12] Rebay, M., and Padet, J., 1999, "Laminar Boundary-Layer Flow Over a SemiInfinite Plate Impulsively Heated or Cooled,” Eur. Phys. J.: Appl. Phys., 7, pp. 263-269.

[13] Kurkal, K. R., and Munukutla, S., 1989, "Thermal Boundary Layer Due to Sudden Heating of Fluid," J. Thermophys. Heat Transfer, 3, pp. 470-472.

[14] Munukutla, S., and Kurkal, K. R., 1988, "Computational Analysis of Unsteady Heat Transfer in a Pulsed High Energy Laser Flow Loop," Paper No. AIAA88-2745.

[15] Li, H., and Nalim, M. R., 2007, "Thermal Boundary Layer Response to FarField Flow Temperature Transitions," IMECE Paper No. 2007-41288.

[16] Keller, H. B., 1978, "Numerical Methods in Boundary-Layer Theory," Annu. Rev. Fluid Mech., 10, pp. 417-433.

[17] Cimbala, J. M., 1980, "Fourth-Order Keller Box Solution of the Incompressible Axisymmetric Boundary Layer Equations," Paper No. AIAA-80-0864.

[18] Keller, H. B., and Cebeci, T., 1972, "Accurate Numerical Methods for Boundary-Layer Flows. II: Two-Dimensional Turbulent Flows,” AIAA J., 10, pp. 1193-1199.

[19] Cebeci, T., Khattab, A. A., and Stewartson, K., 1980, "Studies on ThreeDimensional Boundary Layers on Bodies of Revolution. II. ThreeDimensional Laminar Boundary Layers and the OK of Accessibility," Douglas Aircraft Co., Long Beach, CA, Report No. MDC J8716.

[20] Vadyak, J., and Hoffman, J. D., 1984, "Three-Dimensional Flow Simulations for Supersonic Mixed-Compression Inlets and Incidence," AIAA J., 22, pp. 873-881.

[21] Cebeci, T., 1977, "Calculation of Unsteady Two-Dimensional Laminar and Turbulent Boundary Layers With Fluctuations in External Velocity," Proc. R. Soc. London, Ser. A, 355, pp. 22-238.

[22] Rebay, M., Padet, J., and Kakac, S., 2007, "Forced Convection From a Microstructure on a Flat Plate," Heat Mass Transfer, 43, pp. 309-317.

[23] Rees, D. A. S., 1997, "Three-Dimensional Free Convection Boundary Layers in Porous Media Induced by a Heated Surface With Spanwise Temperature Variations," ASME J. Heat Transfer, 119, pp. 792-798.

[24] Ishak, A., Nazar, R., and Pop, I., 2007, "Mixed Convection on the Stagnation Point Flow Toward a Vertical, Continuously Stretching Sheet," ASME J. Heat Transfer, 129, pp. 1087-1090.

[25] Cebeci, T., and Bradshaw, P., 1984, Physical and Computational Aspects of Convective Heat Transfer, Springer, Berlin.

[26] Schlichting, H., and Gersten, K., 2001, Boundary Layer Theory, SpringerVerlag, New York

[27] White, F. M., 1991, Viscous Fluid Flow, McGraw-Hill, New York.

[28] Telionis, D. P., 1981, Unsteady Viscous Flow, Springer-Verlag, New York.

[29] Smith, S. H., 1972, "On the Impulsive Flow of a Viscous Liquid Past a SemiInfinite Flat Plate," SIAM J. Appl. Math., 22, pp. 148-154.

[30] Paxson, D. E., 1995, "A Comparison Between Numerically Modelled and Experimentally Measured Loss Mechanisms in Wave Rotors," J. Propul. Power, 11, pp. 908-914. 